\section{Expanding noninvasive prenatal testing to include microdeletions and segmental aneuploidy: cause for concern?}

To the Editor: We read with great interest the article by Yatsenko et al., " "Maternal Cell-Free DNA-Based Screening for Fetal Microdeletion and the Importance of Careful Diagnostic Follow-Up," describing a single prenatal case identified by noninvasive prenatal screening as harboring a deletion at 22q11.2. Upon diagnostic postnatal testing, however, a smaller, noncritical deletion at 22q11.21 was identified. As stated by the authors, noninvasive prenatal screening (also referred to as noninvasive prenatal testing (NIPT)) has emerged as a powerful tool in screening for fetal aneuploidies. Major providers of this technology have expanded their test offerings to include screening for common microdeletion syndromes. Despite high sensitivity and specificity for common trisomies, the recent literature suggests a need for extreme caution in interpreting NIPT because of false-positive rates higher than previously reported when compared with invasive testing as well as concerns regarding the potential for overrepresentation of the positive predictive value for specific aneuploidies. ${ }^{1,2}$

We wanted to make an important and substantial addition to the observation by Yatsenko et al. ${ }^{3}$ based on our analysis of cases evaluated by NIPT over the past year and subsequently referred to our laboratory for diagnostic testing by chromosomal microarrays and/or karyotype analysis. From our analysis of 287 consecutive samples, NIPT results were available for 278 cases, and diagnostic testing results were available for 277 of these cases. Of the 88 cases with normal NIPT results, diagnostic testing was concordant (normal chromosomal microarrays and/or karyotype) in 79 cases; discordant in 4 ( 2 cases with gender discordance, 1 case with monosomy $\mathrm{X}$, and 1 case with two large duplications at 1q42.13q44 and 22q11.1q12.3); and concordant but with a structural variation in 5 cases (1 case with inversion 7, 3 cases with a small deletion or duplication, 1 case with a deletion and a duplication). For 162 cases with NIPT suggestive of a whole-chromosome aneuploidy, diagnostic testing was concordant in $69.8 \%$ cases, with the highest true-positive rate for trisomy 21 (88.4\%) and the lowest for monosomy X (28\%). Results were discordant in $23.5 \%$ of cases, with the highest discordant rates for trisomy 13 and monosomy X (47 and 72\%, respectively). In 11 cases (6.7\%), diagnostic testing revealed results that were only partially concordant with NIPT.

The most significant and recent observation we wish to highlight in this letter is 25 cases for which NIPT results suggested a microdeletion or a segmental aneuploidy (Table 1). The falsepositive rates were uniformly high for the common microdeletions tested for by some providers using expanded versions of NIPT. Notably, diagnostic testing revealed false-positive NIPT results in five of seven cases with 22q11.21 deletion, five of six cases with $5 \mathrm{p}$ deletion, three of four cases with $1 \mathrm{p} 36$ deletion, and one of one case with $4 p$ deletion. Diagnostic testing results

Table 1 Invasive testing results for cases with microdeletions or segmental aneuploidies reported by NIPT

\begin{tabular}{|c|c|c|c|c|}
\hline \multirow[b]{3}{*}{ Abnormal NIPT results (26) } & \multicolumn{4}{|c|}{ Array/karyotype results } \\
\hline & \multirow[t]{2}{*}{ Concordant (6) } & \multirow{2}{*}{$\begin{array}{c}\text { Discordant (17) } \\
\text { No. }\end{array}$} & \multirow{2}{*}{$\begin{array}{c}\text { Description } \\
\text { (discordant results) }\end{array}$} & \multirow[t]{2}{*}{ Concordant + structural abnormality (3) } \\
\hline & & & & \\
\hline $5 p$ Deletion (6) & 1 & 5 & All normal & 0 \\
\hline 1 p36 Deletion (4) & 1 & 3 & All normal & 0 \\
\hline 15q Deletion (1) & 1 & 0 & & \\
\hline 9p Duplication (1) & 0 & 0 & & 1 (idic 9p) \\
\hline 13q Deletion (1) & 1 & 0 & & \\
\hline 18p Deletion $+18 q$ deletion $(1)$ & 0 & 1 & $\begin{array}{l}\text { 14-Mb deletion: } \\
\text { 18p11.32p11.21 }\end{array}$ & \\
\hline $\begin{array}{l}\text { No result for chromosome } \\
13 \text { (1) }\end{array}$ & 0 & 1 & $\begin{array}{c}\text { ROH chromosome } \\
\text { 13/?UPD13 }\end{array}$ & \\
\hline
\end{tabular}

idic, isodicentric; NA, data not available; NIPT, noninvasive prenatal testing; ROH, region(s) of homozygosity. 
were concordant for a single case each where NIPT results identified a $15 \mathrm{q}$ deletion, a $9 \mathrm{p}$ duplication (confirmed to be an isodicentric (9p) duplication), and a 13 q deletion, respectively. In one case where NIPT was suggestive of both $18 \mathrm{p}$ and $18 \mathrm{q}$ terminal deletions, diagnostic testing revealed only a deletion of $14 \mathrm{Mb}$ at $18 \mathrm{p} 11.32 \mathrm{p} 11.21$. In a single case with NIPT suggestive of trisomy 18q, diagnostic testing showed a duplication of $18 \mathrm{p} 11.21 \mathrm{q} 23$ that was further characterized as a translocation of the duplicated segment to the p-arm of one chromosome 13, resulting in trisomy for the 18p11.21q23 segment. In one case where NIPT results indicated a "partial deletion of 21q," diagnostic testing revealed a 9.2-Mb duplication at 21q11.2-q21.1 that was inserted at 14p11.2. In a single case where NIPT results were inconclusive for chromosome 13, chromosomal microarrays showed a large region of allelic homozygosity for chromosome 13.

In conclusion, our results suggest an overall concordance rate between NIPT and invasive/diagnostic testing of $\sim 69 \%$, a discordance rate of $20 \%$, and a partial concordance rate of $11 \%$. The frequency of structural abnormalities that may not be defined or are poorly defined by NIPT is substantial. In addition, the ambiguous and nonspecific nature of NIPT results introduces a significant degree of confusion for clinicians and patients alike. The cases listed in this report and those from recent publications warrant additional large studies to determine the sensitivity and specificity of current NIPT technologies to detect microdeletions, with an explicit definition of breakpoints. Until now, identification of clinically relevant unbalanced genomic alterations, specifically microdeletions and microduplications, has been driven primarily by diagnostic testing and has been precluded from any screening tests. With the multiple layers of complexity associated with pathogenic copy-number alterations, such as variability in size, gene content, variable penetrance and expressivity, and genetic and phenotypic heterogeneity, it would be extremely prudent to take a much more cautious approach to expanding NIPT/noninvasive prenatal screening for microdeletions. It is our belief that, unlike wholechromosome aneuploidies, biological causes such as confined placental mosaicism may not play as large a role in contributing to the high false-positive rates for microdeletions/microduplications. As has been recommended, extensive pretest genetic counseling with a complete discussion of the benefits and limitations of NIPT versus diagnostic testing must occur, specifically for low-risk patients with abnormal NIPT and high-risk patients with normal NIPT.

\section{DISCLOSURE}

The authors are employees of CombiMatrix Diagnostics and T.S., K.H., and N.D. own stock in the company.

Trilochan Sahoo, $M D^{1}$, Karine Hovanes, $P h D^{1}$, Michelle N. Strecker, $M S^{1}$, Natasa Dzidic, $M S^{1}$, Sara Commander, $M S^{1}$ and Mary K. Travis, $M S^{1}$

${ }^{1}$ Combimatrix Molecular Diagnostics, Irvine, California, USA. Correspondence: Trilochan Sahoo (tsahoo@combimatrix.com)

\section{REFERENCES}

1. Cheung SW, Patel A, Leung TY. Accurate description of DNA-based noninvasive prenatal screening. N Engl J Med 2015;372:1675-1677.

2. Wang JC, Sahoo T, Schonberg S, et al. Discordant noninvasive prenatal testing and cytogenetic results: a study of 109 consecutive cases. Genet Med 2015;17:234-236.

3. Yatsenko SA, Peters DG, Saller DN, Chu T, Clemens M, Rajkovic A. Maternal cellfree DNA-based screening for fetal microdeletion and the importance of careful diagnostic follow-up. Genet Med 2015;17:836-838.

Advance online publication 21 January 2016. doi:10.1038/gim.2015.196 